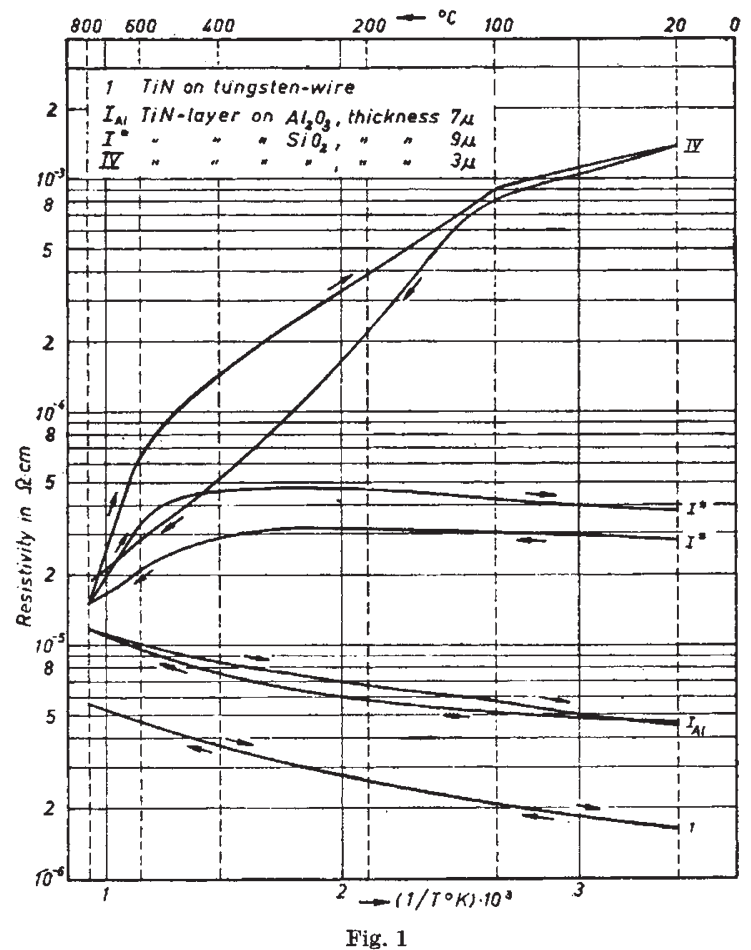

has exactly the same value in both cases $(4 \cdot 2346 \pm$ $0.0002 \mathrm{~A}$.). Fig. 1 shows the relation of the specific resistivity to temperature for typical samples. Some numerical results are given in Table 1. Essentially the same behaviour has been observed for titanium carbide and mixed crystals of titanium nitride and carbide.

A full description of the experiments and the theoretical discussion will be published elsewhere.

A. MÜNSTER

K. SAGEL

G. Schlamp

Metals Laboratory,

Metallgesellschaft A.G.,

Frankfurt (Main). Aug. 30.

${ }^{1}$ Cf. "Gmelins. Handbuch der anorganischen Chemie", vol. "Titan" 2 Van Arkel, A. E., Physica, 4, 286, 293 (1924).

\section{Point Source and Sink in a Parallelepiped}

THE potential distribution in a parallelepiped arising from a point source and sink is of practical importance in the measurement of the thickness of plates and tubes ${ }^{1,2}$, and the electrical conductivity of semiconductors ${ }^{3}$. A common practical arrangement employs four collinear equi-spaced contacts : current $I$ is passed between the outer pair, and the potential difference $V$ between the inner pair is measured. If the contacts are aligned centrally parallel to the longest side $2 \alpha$ of a rectangular block of material, its width being $2 \beta$ and its thicknoss $\gamma$ in units of the electrode spacing, the conductivity is given by the triple series :

$\sigma=\frac{I}{\pi V} \sum_{-\infty}^{\infty} \sum_{-\infty}^{\infty} \sum_{-\infty}^{\infty}(-1)^{n}\left\{\left[(2 n \alpha+1)^{3}+4 m^{2} \beta^{2}+4 r^{2} \gamma^{2}\right]^{-1 / 2}\right.$

$$
\left.-\left[(2 n \alpha+2)^{2}+4 m^{2} \beta^{2}+4 r^{2} \gamma^{2}\right]^{-1 / 2}\right\}
$$

The difficulty in computing this series lies in the slow convergence of the single series :

$$
s=\sum_{-\infty}^{\infty}\left[\left(n^{2}+x^{2}\right)^{-1 / 2}-\left(n^{2}+y^{2}\right)^{-1 / 2}\right]
$$

when $x$ and $y$ are large. Warren ${ }^{2}$ applied the EulerMaclaurin summation formula to obtain approximations, and other workers have offered no alternative formula.

Although it is not difficult to show that

$$
s=\int_{-\infty}^{\infty}\left[\frac{H_{0}^{(1)}(x t)-H_{0}^{(1)}(y t)}{\varepsilon^{t}-1}\right] \mathrm{d} t,
$$

and thence to obtain the alternative expansion

$$
s=\left\{2 \log (y / x)+4, \sum_{n=1}^{\infty}\left[K_{0}(2 \pi n x)-K_{0}(2 \pi n y)\right]\right\},
$$

this result does not appear to have been published hitherto.

It is a simple matter to reduce triple summations to a calculable form by means of this transformation, and the result may be readily extended to series involving higher inverse powers of $\left(n^{2}+x^{2}\right)$ and $\left(n^{2}+y^{2}\right)$. Such series are important in solid-state crystal theories, where they have usually led to tedious approximations, although recently a more powerful approach has been described ${ }^{4}$.

A. C. Sn

L. LEWIN

Standard Telecommunication Laboratories, Ltd., Progress Way,

Great Cambridge Road,

Enfield, Middlesex.

'Thornton, B. M., and Thornton, W. M., Proc. Inst. Mech. Eng., 140, 349 (1938).

Warren, A. G., J. Inst. Elect. Eng., 84, 91 (1939).

'Valdes, L. B., Proc. Inst. Rad. Eng., 42, 420 (1954).

- Van der Hoff, B. M. E., and Benson, G. C., Can. J. Phys., 31, 1087 (1953).

\section{Dielectric Increment of Sodium Alginate as a Function of Polyion and Counter-lon Concentration}

A NUMBER of linear polyelectrolytes - acid as well as basic-give high dielectric increments in aqueous solution, when compared with less asymmetric proteins and simple dipolar ions.

The sodium salt of alginic acid (poly- $\beta$ - $d$-mannuronic acid) has been shown recently to have dielectric properties in aqueous solution similar to the other polyelectrolytes studied, namely, high dielectric increments which show anomalous dependence upon concentration (see Fig. 1), dielectric dispersions at radio-frequencies which are strongly dependent upon concentration, decrease of increments and change of dispersions with addition of small amounts of simple electrolytes, and decrease of increments and change of dispersion with decrease of dielectric constant of solvent (water changed to ethanol-water) ${ }^{1}$. It was thought of interest to investigate in more detail the effect of excess counter ions.

The ellipsoid method ${ }^{2}$, which permits measurements with solutions with comparatively high electric conductivity, was used for the determination of dielectric constants, which were made at various frequencies in the range $30 \mathrm{Mc} / \mathrm{sec}$. to $30 \mathrm{kc} . / \mathrm{sec}$., so that the dispersion curve due to the solute(s) was obtained in each case. From this the dielectric con- 